\title{
Sepsis and ECMO
}

\section{Ram Gopalakrishnan ${ }^{1} \cdot$ Rohit Vashisht $^{1}$}

Received: 19 October 2019 / Revised: 24 February 2020 / Accepted: 26 February 2020 / Published online: 14 May 2020

(C) Indian Association of Cardiovascular-Thoracic Surgeons 2020

\begin{abstract}
Sepsis is being recognized as an important complication of extracorporeal membrane oxygenation (ECMO) and its presence is a poor prognostic marker and increases the overall mortality. The survival rate differs in the various types of cannulation techniques. Adult patients with prolonged duration of ECMO constitute the major risk population. Ventilator-associated pneumonia and bloodstream infections form the main sources of sepsis in these patients. It is important to know the most common etiological agents for sepsis in ECMO, which varies partly with the local epidemiology of the hospitals. A high index of suspicion, drawing adequate volumes for blood culture and early and timely administration of appropriate empirical antimicrobials can substantially decrease the morbidity and mortality in this high-risk population. The dosing of antimicrobials is influenced by the pharmacological variations on ECMO machine and is an important consideration. Infection control practices are of paramount importance and need to be followed meticulously to prevent sepsis in ECMO.
\end{abstract}

Keywords ECMO $\cdot$ Sepsis $\cdot$ Culture $\cdot$ Antimicrobials $\cdot$ Infection control

\section{Introduction}

ECMO can be either veno-venous cannulation (VV), which is used predominantly in hypoxic respiratory failure states like acute respiratory distress syndrome or veno-arterial cannulation (VA), which is the main or preferred modality for cardiac support system like postoperative cardiac surgery and cardiogenic shock. The survival rate for VV-ECMO is $60 \%$ and for VA-ECMO is $45 \%$ when death is imminent [1-4]. Sepsis can be a reason for initiating ECMO, and on the other hand, patients can develop sepsis on ECMO too.

The most frequently encountered complications of ECMO are hemorrhagic, thrombotic, and infective events [5-7]. Among all the complications of ECMO, sepsis stands out prominently. Allyn et al. [1] in their study on complications of ECMO have demonstrated that out of the 145 patients studied, 65 patients (44.8\%) finally developed sepsis. Peitz et al. [8] studied the incidence and characterization of sepsis in

Ram Gopalakrishnan

gopalmeena_2000@yahoo.com

Rohit Vashisht

neharohitvashisht@gmail.com

1 Department of Infectious Diseases, Apollo Hospital, Chennai 600 006, India
ECMO population and showed that out of 89 patients included in the study, $41.6 \%$ developed sepsis after ECMO cannulation. Factors contributing to sepsis include high severity of underlying illness, disease-induced compromised immune systems, and a large number of indwelling medical devices.

\section{Risk factors for sepsis}

The risk factors for developing septic complications in ECMO include:

a. Age: Adult patients who undergo ECMO are at greater risk for developing a nosocomial infection and sepsis than neonates and children $(20.5 \%$ of adults vs. $6.1 \%$ of neonates and pediatric patients develop culture-proven infections during ECMO) [7].

b. Duration of ECMO: The time duration of ECMO support carries a directly proportionate risk of a patient acquiring serious infection during ECMO and is associated with a significantly increased rate of death $[9,10]$. Burket et al. [11] had shown that the bloodstream infection (BSI) rate increased with the duration of ECMO support. The rate of BSI was 9.5 cases per 1000 ECMO-days among patients who underwent ECMO for 3-10 days, 27.2 cases per 1000 ECMO-days and 64.5 cases per 1000 ECMO-days, 
among those who underwent ECMO for 11-20 days and 21-30 days, respectively.

In the study done by Kim et al. [12], the incidence of infection increased proportionately with time on ECMO (Table 1).

c. Presence of foreign devices: Any foreign device serves as a portal of entry for micro-organisms and predisposes to sepsis. In ECMO patients, the presence of intravascular devices, such as central venous catheters, intra-arterial lines, large-bore ECMO cannulae, endotracheal tubes, and intra-aortic balloon pumps, all cause disruption of the protective barrier mechanism of skin and help pathogens gain entry into the human system [13]. In addition, multiple ports on these devices can get contaminated and this adds further to the ongoing risk of acquiring nosocomial sepsis.

d. Comorbidities: Sun et al. [14] have reported an increased risk of infection in patients who had autoimmune comorbidities or who were on a chronic immunosuppressive regimen. Out of the 10 patients with autoimmune disease, 5 developed infections ( $p$ value 0.003 ). The diseases included were systemic lupus erythematosus, dermatomyositis, Wegener's granulomatosis, antiphospholipid syndrome, and scleroderma. It was found that 8 of these patients had received high-dose immunosuppression before initiating ECMO which might have compromised their immunity and predisposed them to sepsis.

e. Cannulation techniques and circuit of ECMO: In the neonatal population, there is a $25 \%$ increased risk of infective complications with the VA-ECMO modality, as compared with the VV-ECMO [15]. Even studies in older children show a similar trend [7,9]. Only one study done by Sun et al. [14] surprisingly found a greater infection risk in VV-ECMO patients when compared with VAECMO, but these results could be due to the more severe underlying medical conditions in VV-ECMO patients in that study. The double-lumen internal jugular cannulae have also been shown to be associated with a decreased risk of infection in the adult population [15]. On the other hand, femoral cannulation may increase the risk of catheter-related bloodstream infection (CR-BSI) and sepsis during ECMO.

f. Severity of illness: A high Sequential Organ Failure Assessment (SOFA) score [16] before cannulation, has been found to be an independent risk factor for sepsis

Table 1 Relation of duration of ECMO with incidence of infection

\begin{tabular}{llllll}
\hline Days of ECMO & $2-3$ & $3-6$ & $6-9$ & $9-12$ & $>12$ \\
\hline Incidence of infection (\%) & 0 & 16 & 23.1 & 60 & 100 \\
\hline
\end{tabular}

especially in adults. The overall SOFA score was an average of 10.6 in the infected group versus 8.3 in the noninfected group $(p=0.030)$ [17] and was statistically significant for BSIs too (on average 13 in the BSI group versus 12 in the non-BSI group; $p=0.038$ ) [7].

\section{Effect of sepsis on mortality}

Sepsis increases mortality in the ECMO population. Meyer et al. [18] demonstrated that development of sepsis was a bad prognostic sign and an independent negative predictor of survival in the neonatal population. Moreover, in this study, septic patients had a significantly higher frequency of seizures, gastrointestinal hemorrhage, metabolic complications (like hypoglycemia, hypernatremia), mechanical problems (like hemofilter malfunction, clots in the ECMO circuit), and a greater need for red blood cell transfusion [18].

\section{Possible sources of sepsis in ECMO patients}

Potential sources of sepsis in ECMO patients include ventilator-associated pneumonia (VAP), BSI, cannula infections, mediastinitis, catheter-associated urinary tract infections (CA-UTI), infected pressure sores, and Clostridium difficileassociated diarrhea. However, pneumonia and BSI constitute the majority of infections. Schmidt et al. [5] studied 220 patients who underwent ECMO support (VA-ECMO) for $>48 \mathrm{~h}$ and found that $142(64 \%)$ developed nosocomial infections. The relative incidence of VAP, BSI, cannula infections, and mediastinitis was found to be $55 \%, 18 \%, 10 \%$, and $11 \%$ of the patients, respectively. The majority of patients with VAP had secondary bacteremia. Septic shock was secondary to VAP $(36 \%)$, mediastinitis $(35 \%)$, and bloodstream infections $(43 \%)$, with a smaller contribution from cannula (19\%) and catheter-related $(20 \%)$ infections.

A recent study was done by $\mathrm{Kim}$ et al. [12], where 61 patients with cardiogenic shock underwent VA-ECMO. There were 18 nosocomial infection events in $14(23.0 \%)$ patients. BSI were seen in 9 cases, and respiratory tract infection (RTI) was found in 9 cases. There was no urinary tract infection or surgical site wound infection in this study. In the study by Sun et al. [14], out of the 55 episodes of infection, only 3 were urinary tract infections.

\section{Causative agents of sepsis in ECMO}

As per the records of extracorporeal life support organization (ELSO) registry, the most frequent infective agent during ECMO is coagulase-negative staphylococcus (CoNS). The 
other important etiological agents are Candida species (sp.), Pseudomonas aeruginosa, Enterobacteriaceae, Staphylococcus aureus, and Enterococcus sp. [9].

Previous reports have mentioned that CoNS are the most frequent causative agents of BSI accounting for up to $37 \%$ in studies up to 2006. This could represent an over-estimation of the role of CoNS because of the misleading diagnostic criteria for BSI and CR-BSI in these studies. In more recent studies, CoNS were less frequent causes of BSIs (approximately $4 \%$ of total cases) $[17,19,20]$.

Candida spp. are another common and important pathogen causing infections during ECMO support [11, 13, 17, 21]. It has also been implicated as an agent of bloodstream infectionrelated sepsis accounting on average for $13 \%$ of BSIs. ECMO patients are already at high risk for disseminated candidiasis in view of being critically ill, having multiple central lines including ECMO cannulation, prolonged exposure to broad spectrum antibiotics, and getting total parenteral nutrition [22].

The ECMO cannulae and membrane oxygenators can be colonized with CoNS and Candida spp. with subsequent BSI [23]. Gram-negative bacteria cause on an average of $31 \%$ of the reported cases of VAP and $16 \%$ of the BSI in various studies. On the other hand, Staph aureus has been seen in $10 \%$ of VAP cases and $7 \%$ of the BSI-related sepsis [24]. The causative micro-organisms associated with various infections in study done by Schmidt et al. [5] are depicted in (Table 2).

The urinary isolates found in the study by Sun et al. [14] were E. coli (1/3) and Candida albicans (2/3).

In a recent study by Kim et al. [12], Gram-negative pathogens were predominant in both RTI and BSI. Acinetobacter baumannii ( $n=8,88.9 \%$ ) was the most common respiratory tract pathogen in this study. In terms of BSI, A. baumannii $(n=2)$, and Pseudomonas aeruginosa $(n=2)$ were the most common pathogens. Local epidemiology of hospitals, different study designs, differences in defining sepsis criteria in
ECMO patients, and different prevention policies could be the reasons for varying prevalence of different microbes.

As far as we know, there is no available Indian data or studies revealing the etiological agents of sepsis specifically in ECMO. It is to be noted that Indian intensive care units (ICUs) report a much greater prevalence of Gram-negative organisms, compared with the international literature $[25,26]$.

\section{When is sepsis to be suspected in an ECMO patient?}

Clinical scoring like Acute Physiologic Assessment and Chronic Health Evaluation (APACHE) [27] and SOFA scores [16] have been given importance now in early identification of sepsis. As per the Sepsis 3 definitions, acute change in total SOFA score of at least 2 points indicates sepsis and organ dysfunction [28].

Allyn et al. [1] in their study defined sepsis as occurrence of a documented nosocomial infection such as pneumonia, bacteremia, catheter-derived infections, and surgical site infections or the occurrence of septic shock as per the Sepsis-3 guidelines [28]. However, in another study by Peitz et al. [8], they used the systemic inflammatory response syndrome (SIRS) criteria for defining any new septic event.

A high index of suspicion is very important to detect early sepsis and reduce mortality in these high-risk patients. The most important challenge is to differentiate clinically between sepsis and SIRS [29], as the latter is common in these patients because of underlying cardiac or pulmonary insult and also due to complex circuits of ECMO. Both clinical examination and biomarkers play an important role. It is important to note that the critically ill patients may have some degree of baseline hyperthermia which always does not denote infection [30]. However, patients on ECMO, who are able to produce fever

Table 2 Microorganisms associated with various nosocomial infections in 142 extracorporeal membrane oxygenation patients

\begin{tabular}{|c|c|c|c|c|c|c|c|}
\hline \multicolumn{2}{|c|}{ Ventilator-associated pneumonia } & \multicolumn{2}{|l|}{ Cannula infections } & \multicolumn{2}{|c|}{ Post-sternotomy mediastinitis } & \multicolumn{2}{|c|}{ Bloodstream infection } \\
\hline \multicolumn{2}{|l|}{$n=163$} & \multicolumn{2}{|l|}{$n=21$} & \multicolumn{2}{|l|}{$n=23$} & \multicolumn{2}{|l|}{$n=47$} \\
\hline Organism & $\operatorname{No}(\%)$ & Organism & $\operatorname{No}(\%)$ & Organism & $\operatorname{No}(\%)$ & Organism & $\mathrm{No}(\%)$ \\
\hline Pseudomonas & $43(26)$ & E. coli & $5(24)$ & Candida sp. & $8(35)$ & Pseudomonas & $10(21)$ \\
\hline Polymicrobial & $19(12)$ & Enterococcus sp. & $4(19)$ & S. epidermidis & $7(30)$ & Enterococcus sp. & $7(15)$ \\
\hline Staph aureus & $16(10)$ & S. epidermidis & $4(19)$ & Pseudomonas & $2(9)$ & E. coli & $6(13)$ \\
\hline Enterobacter sp. & $16(10)$ & Polymicrobial & $4(19)$ & Staph aureus & $2(9)$ & S. epidermidis & $5(10)$ \\
\hline E. coli & $14(9)$ & Staph aureus & $2(10)$ & E. coli & $2(9)$ & Staph aureus & $4(9)$ \\
\hline H. influenza & $14(9)$ & Pseudomonas & $2(10)$ & Enterobacter sp. & $1(4)$ & Streptococcus sp. & $3(6)$ \\
\hline Klebsiella sp. & $10(6)$ & Proteus & $1(5)$ & Neisseria sp. & $1(4)$ & Enterobacter sp. & $3(6)$ \\
\hline Neisseria sp. & $5(3)$ & & & & & Candida sp. & $3(6)$ \\
\hline Proteus mirabilis & $5(3)$ & & & & & Anaerobes & $3(6)$ \\
\hline
\end{tabular}

n, number; No(\%), percentage number; sp., species; E. coli, Escherichia coli; H. influenza, Hemophilus influenza; S. epidermidis, Staph epidermidis 
of at least 101 degrees or more are likely to have a high inflammatory response and should be closely monitored [31] for other signs of infection. Patients also should be monitored for any new hypothermia, hemodynamic instability, increased respiratory distress with desaturation, purulent tracheal secretions, frank pyuria or fall in urine output requiring renal replacement therapy, alteration in sensorium, coagulopathy, and new skin lesions (purpura fulminans). All these clinical pointers may indicate sepsis and call for timely intervention.

Laboratory parameters such as leukocytosis and leucopenia are not very accurate in ECMO patients [31]. A retrospective analysis in neonates failed to demonstrate predictive value of white blood cell (WBC) counts for nosocomial infections in patients on ECMO [32]. In the same way, thrombocytopenia is also to be interpreted with caution as it is fairly common due to the activation by ECMO circuits [31]. Inflammatory markers like C-reactive protein (CRP) are important sensitive tools in diagnostic evaluation but they are not specific. Serum procalcitonin (PCT) has been documented as a more specific biomarker of systemic infection and sepsis than the previous conventional markers like WBC count and CRP [33]. Pieri et al. [34] showed in his study that in patients who were receiving VA-ECMO, PCT has good accuracy with the cutoff of $1.89 \mathrm{ng} / \mathrm{mL}$ (sensitivity $87.8 \%$, specificity $50 \%$ ) and CRP having cutoff of $97.70 \mathrm{mg} / \mathrm{L}$ is $85.3 \%$ sensitive and $41.6 \%$ specific. However, PCT was not found to be a reliable marker of sepsis in VV-ECMO in this study. The PCT and CRP as a combined assay had a very high overall sensitivity of $87.2 \%$, though specificity was $25.9 \%$. Another study was done by Tanaka et al. [35], where he studied the effectiveness of PCT in differentiating infection from SIRS in patients on ECMO and found that PCT with a cutoff of 2 had a sensitivity of $90 \%$ and specificity of $82 \%$ with a positive predictive value of $82 \%$ and negative predictive value of $90 \%$. The better specificity in this study was hypothesized to be due to exclusion of PCT assays after prolonged systemic malperfusion. Nevertheless, PCT has to be interpreted with caution as elevated levels can be found in situations of massive stress like post trauma, post-surgery, severe cardiogenic shock, and compromised renal function [36]. Hence, PCT should not be used as an isolated test for diagnosis of sepsis, and it preferably should be clinically correlated using serial values to see a rising or falling trend. It has greater value in de-escalation of antibiotics in sepsis in ICU patients [37] and this can be easily extrapolated for ECMO patient also.

\section{Workup of suspected sepsis in the ECMO patient}

Patients with suspected sepsis on clinical grounds require routine tests including complete blood counts, liver and kidney function tests, urine analysis, and chest X-ray. Cultures play a very important role. Two sets of blood cultures ( 4 bottles,
$10 \mathrm{ml}$ of blood per bottle, $10-30 \mathrm{~m}$ apart) and a urine culture are to be drawn at first suspicion of sepsis [38]. Endotracheal (ET) cultures are to be sent if there is clinical evidence of VAP, e.g., a high Clinical Pneumonia Index Scale (CPIS).

Drawing a serum procalcitonin at baseline and serial measurements later to see the trend may be helpful. Like in any critically ill patient on prolonged mechanical ventilation with central lines and antibiotic exposure, one should always think of disseminated candida in case of any new sepsis event and sterile cultures and no other obvious focus of infection. If disseminated candidiasis is a possibility, drawing blood for serum beta-D-glucan (BDG) assay at the time of blood cultures may be useful in the diagnosis [39]. A flowchart for proposed workup of sepsis in ECMO is illustrated in (Fig. 1).

\section{Role of antibiotic prophylaxis in ECMO}

There is no data to support the use of routine antibiotic prophylaxis in ECMO patients [31].

Prophylaxis will increase the chance of emergence of resistance strains of microbes and predispose to complications like disseminated candidiasis. If transthoracic cannulation of ECMO is done through open chests in cardiac patients, then these patients are at high risk of developing mediastinitis [13, 40]. In these cases, short course of antibiotic prophylaxis may be considered on a case to case basis. For cannulation purpose, prophylactic antibiotics should be as per standard guidelines for surgical prophylaxis and a single dose is sufficient for percutaneous cannulation techniques.

\section{Empirical antimicrobial selection in the ECMO patient}

In general, treatment of sepsis after ECMO cannulation should follow the same principles as with ICU patients who are not on ECMO. As per the ELSO database and Indian epidemiology, the most important microbes grown from the blood include CoNS, Acinetobacter, Enterobacteriaceae, Pseudomonas, Staph aureus, and Candida; hence, the empiric therapy can be directed to these organisms.

The choice of empiric antibiotic regimen has to be made based on the local epidemiological factors and hospitals' own antibiograms. India has high rates of Gram-negative resistance in tertiary care settings. The Indian council of medical research (ICMR) data in 2015 showed that carbapenem resistance among the Gram-negative bacteria was very high (highest being for A. baumannii followed by $K$. pneumoniae, $P$. aeruginosa, and E. coli). In addition to Gram-positive coverage with drugs such as vancomycin, it becomes prudent to broaden cover by adding high-end antibiotics such as carbapenems, polymyxins, and/or fosfomycin especially in hemodynamically 


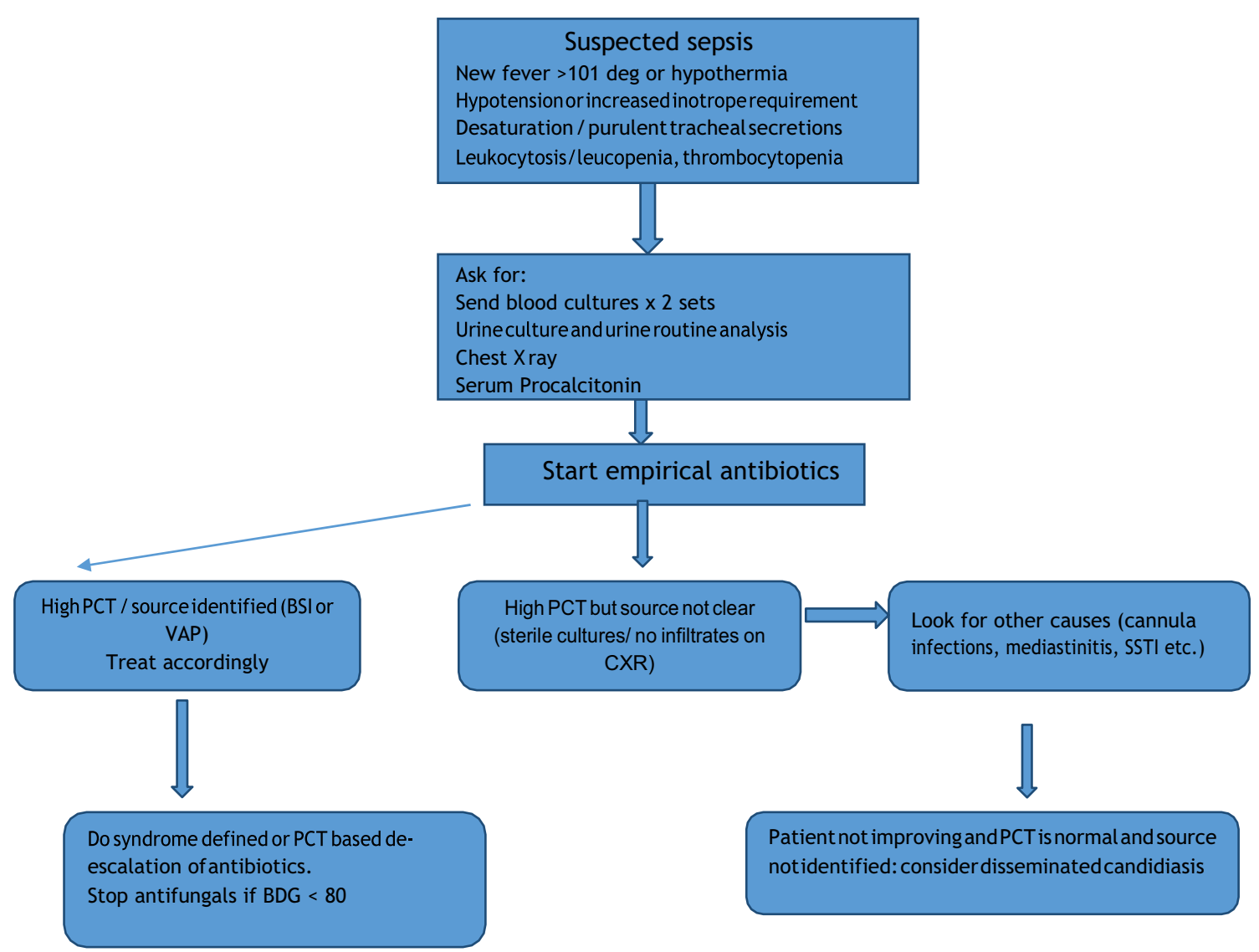

Fig. 1 Algorithm for sepsis workup

unstable patients. Usually these highly critical patients warrant combination therapy initially for control of sepsis which can be de-escalated later based upon the culture sensitivity reports.

Regarding empirical candida coverage, the ESLO infectious disease task force strongly recommends that in view of high incidence and high mortality of candida sepsis in ECMO subset, clinicians should keep the threshold low for starting antifungals in these patients. However, recently Quentin de Roux et al. [41] showed in his results that candidemia occurred in the third week after VA-ECMO implantation and hence antifungal therapy should not be added upfront in empirical regimen in the first 2 weeks of ECMO-related septic shock. Nevertheless, the decision regarding antifungals should be supported by clinical judgement and individualized as per patient needs. Echinocandins remain the class of choice in the hemodynamically unstable patients and this stands true for ECMO population too.

\section{Pharmacokinetic/pharmacodynamics and antimicrobial dosing in ECMO}

Another important point to be noted in ECMO patients is that pharmacokinetic/pharmacodynamics (PK/PD) changes during extracorporeal circuit [42]. The various mechanisms by which this happens are mentioned below:

a. Direct extraction by the ECMO circuit: The highly lipophilic and protein-bound drugs are extracted more through the circuit as demonstrated in ex vivo studies [43-45].

b. Altered clearance of the drugs: Alteration in kidney function is common in ECMO and can increase the chance of drug toxicity of those antibiotics which are primarily excreted through renal route.

c. Changes in volume of distribution: ECMO is known to increase the volume of distribution of certain drugs because the inflammatory response induced by ECMO circuit results in capillary leakage and edema which can increase the volume of distribution $[46,47]$.

Vancomycin is the most common drug studied for PK/PD variations in ECMO [48]. Various studies show an increased volume of distribution and decreased clearance for vancomycin for neonates and infants on ECMO [49]. However, no variations have been seen in the PK/PD parameters of drugs like piperacillin-tazobactam, meropenem, and amikacin. However, most of this data on PK/PD has been derived from the neonatal population and dosing recommendations for adults remain mostly unchanged as ECMO has little effect 
Table 3 Dosing of antimicrobials in ECMO

\begin{tabular}{|c|c|c|c|}
\hline Drug & Volume of distribution & Clearance & Dosing recommendations \\
\hline Vancomycin & $\begin{array}{l}\text { Adult: Unchanged } \\
\text { Neonates: Increased }\end{array}$ & $\begin{array}{l}\text { Adults: Unchanged } \\
\text { Neonates: Decreased }\end{array}$ & $\begin{array}{l}\text { Adults: Standard dosing } \\
\text { Neonates: } 20 \mathrm{mg} / \mathrm{kg} \text { once and then check TDM at } 2 \text { and } \\
8-12 \mathrm{~h} \text { concentrations }\end{array}$ \\
\hline Meropenem & $\begin{array}{l}\text { Adult: Unchanged } \\
\text { Neonates: Not reported }\end{array}$ & $\begin{array}{l}\text { Adult: Unchanged } \\
\text { Neonates: Increased }\end{array}$ & $\begin{array}{l}\text { Adults: } 1 \mathrm{~g} \text { q } 8 \text { hourly for susceptible organisms }(\mathrm{MIC}<2) \\
\text { Neonates: } 40 \mathrm{mg} / \mathrm{kg} \text { loading dose then } 200 \mathrm{mg} / \mathrm{kg} / \text { day } \\
\text { continuous infusion }\end{array}$ \\
\hline Ceftriaxone & Neonates: Increased & Neonates: Unchanged & Neonates: Standard dosing \\
\hline Piperacillin-tazobactam & Adults: Unchanged & Adults: Unchanged & Adults: Standard dosing \\
\hline Tigecycline & Adults: Unchanged & Adults: Unchanged & Adults: Standard dosing \\
\hline Caspofungin & Neonates: Increased & Neonates: Increased & $>78 \mathrm{mg} / \mathrm{m}^{2}$ daily \\
\hline Liposomal amphotericin B & Adults: Not reported & Adults: Not reported & Standard dosing \\
\hline Oseltamivir & Adults: Unchanged & Adults: Unchanged & Standard dosing \\
\hline
\end{tabular}

$T D M$, therapeutic drug monitoring; $M I C$, minimum inhibitory concentration

on their volume of distribution and clearance kinetics [42]. Dosing of antimicrobials commonly used in ICU in ECMO [42] is shown in (Table 3).

\section{Infection control practices and prevention of sepsis in ECMO}

Infection control is an integral part in the management of sepsis in ECMO patients. The extracorporeal life support organization has formulated certain guidelines [31] which should be meticulously followed in each ECMO equipped centers.

a) The first and the foremost is hand hygiene which is the cornerstone of infection control [50].

b) The ECMO circuit is just like a protected central line and no routine sampling to be done from the circuit lines to avoid any kind of contamination.

c) Only continuous infusions such as inotropes and vasopressors should be administered through the ECMO circuit so that sterility of lines is not interrupted as happens in intermittent infusions.

d) Needleless hubs and other sterile practices for all connections are recommended as they are sterilized more reliably with the prep solutions and are safe to users.

e) Chlorhexidine should be used as the agent of disinfection rather than povidone iodine or alcohol.

f) Strict contact isolation should be followed for other patients harboring resistant organisms, who should preferably be spatially separated from patients on ECMO.

g) The VAP prevention bundle interventions include elevation of head of bed to 30-45, daily assessing the readiness to extubate, prophylaxis for peptic ulcer disease, etc. Adult patients should be considered for early tracheostomy. h) Prolonged total parenteral nutrition should be discouraged in these patients and efforts to be made for early initiation of enteral feeds so as to improve the integrity of gut and to prevent future risk of gut translocation [51].

i) Tunneled catheters for long-term intravenous access are usually not preferred in ECMO subset due to hematoma risk which may get secondarily infected.

j) Daily check to remove central lines and urinary catheters minimizes the risk of central line associated bloodstream infections (CLABSI) and CA-UTI once the patient has been stabilized on ECMO.

\section{Conclusion}

To summarize, sepsis is an important complication of ECMO. A high index of suspicion, drawing adequate volumes for blood culture and early and timely administration of appropriate empirical antimicrobials can substantially decrease the morbidity and mortality in this high-risk population. Infection control practices are of paramount importance and need to be followed meticulously to prevent sepsis in ECMO.

Funding There was no funding received for the review from any source.

\section{Compliance with ethical standards}

Conflict of interest The authors declare that they have no conflict of interest.

Informed consent Not required being a Review Article.

Ethical clearance Not required being a Review Article.

Human and animal rights statement Not required being a Review Article. 


\section{References}

1. Allyn J, Ferdynus C, Lo Pinto HL, et al. Complication patterns in patients undergoing venoarterial extracorporeal membrane oxygenation in intensive care unit: multiple correspondence analysis and hierarchical ascendant classification. PLoS One. 2018;13: e0203643.

2. Sy E, Sklar MC, Lequier L, Fan E, Kanji HD. Anticoagulation practices and the prevalence of major bleeding, thromboembolic events, and mortality in venoarterial extracorporeal membrane oxygenation: a systematic review and meta-analysis. J Crit Care. 2017;39:87-96.

3. Vaquer S, de Haro C, Peruga P, Oliva JC, Artigas A. Systematic review and meta- analysis of complications and mortality of venovenous extracorporeal membrane oxygenation for refractory acute respiratory distress syndrome. Ann Intensive Care. 2017;7:51.

4. Xie A, Phan K, Tsai YC, Yan TD, Forrest P. Venoarterial extracorporeal membrane oxygenation for cardiogenic shock and cardiac arrest: a meta-analysis. J Cardiothorac Vasc Anesth. 2015;29:63745 .

5. Schmidt $\mathrm{M}$, Brechot $\mathrm{N}$, Hariri $\mathrm{S}$, et al. Nosocomial infections in adult cardiogenic shock patients supported by venoarterial extracorporeal membrane oxygenation. Clin Infect Dis Off Publ Infect Dis Soc Am. 2012;55:1633-41.

6. Abrams D, Brodie D. Extracorporeal membrane oxygenation for adult respiratoryfailure. Chest. 2017;152:639-49.

7. Aubron $\mathrm{C}$, Cheng AC, Pilcher D, et al. Infections acquired by adults who receive extracorporeal membrane oxygenation: risk factors and outcome. Infect Control Hosp Epidemiol. 2013;34:24-30.

8. Peitz G, Brown T, Murry DJ. Incidence and characterization of sepsis in an ECMO population. Crit Care Med. 2018;46:325. https://doi.org/10.1097/01.ccm.0000528693.63916.74.

9. Bizzarro MJ, Conrad SA, Kaufman DA, Rycus P. Infections acquired during extracorporeal membrane oxygenation in neonates, children, and adults. Pediatr Crit Care Med. 2011;12:277-81.

10. Schutze GE, Heulitt MJ. Infections during extracorporeal life support. J Pediatr Surg. 1995;30:809-12.

11. Burket JS, Bartlett RH, Vander Hyde K, Chenoweth CE. Nosocomial infections in adult patients undergoing extracorporeal membrane oxygenation. Clin Infect Dis. 1999;28:828-33.

12. Kim GS, Lee KS, Park CK, et al. Nosocomial infection in adult patients undergoing venoarterial extracorporeal membrane oxygenation. J Korean Med Sci. 2017;32:593-8.

13. O’Neill JM, Schutze GE, Heulitt MJ, Simpson PM, Taylor BJ. Nosocomial infections during extracorporeal membrane oxygenation. Intensive Care Med. 2001;27:1247-53.

14. Sun HY, Ko WJ, Tsai PR, et al. Infections occurring during extracorporeal membrane oxygenation use in adult patients. J Thorac Cardiovasc Surg. 2010;140:1125-32.

15. Vogel AM, Lew DF, Kao LS, Lally KP. Defining risk for infectious complications on extracorporeal life support. J Pediatr Surg. 2011:46:2260-4.

16. Vincent JL, Moreno R, Takala J, et al. The SOFA (Sepsis-related Organ Failure Assessment) score to describe organ dysfunction/ failure. On behalf of the Working Group on Sepsis-Related Problems of the European Society of Intensive Care Medicine. Intensive Care Med. 1996;22:707-10.

17. Pieri M, Agracheva N, Fumagalli L, et al. Infections occurring in adult patients receiving mechanical circulatory support: the twoyear experience of an Italian national referral tertiary care center. Med Int. 2013;37:468-75.

18. Meyer DM, Jessen ME, Eberhart RC. Neonatal extracorporeal membrane oxygenation complicated by sepsis. Extracorporeal life support organization. Ann Thorac Surg. 1995;59:975-80.
19. Hsu MS, Chiu KM, Huang YT, Kao KL, Chu SH, Liao CH. Risk factors for nosocomial infection during extracorporeal membrane oxygenation. J Hosp Infect. 2009;73:210-6.

20. Tse-Chang A, Midodzi W, Joffe AR, Robinson JL. Infections in children receiving extracorporeal life support. Infect Control Hosp Epidemiol. 2011;32:115-20.

21. Coffin SE, Bell LM, Manning M, Polin R. Nosocomial infections in neonates receiving extracorporeal membrane oxygenation. Infect Control Hosp Epidemiol. 1997;18:93-6.

22. Blumberg HM, Jarvis WR, Soucie JM, et al. Risk factors for candidal bloodstream infections in surgical intensive care unit patients: the NEMIS prospective multicenter study. Clin Infect Dis. 2001;33: $177-86$.

23. Kuehn C, Orszag P, Burgwitz K, et al. Microbial adhesion on membrane oxygenators in patients requiring extracorporeal life support detected by a universal rDNA PCR test. ASAIO J. 2013;59:368-73.

24. Biffi S, Di Bella S, Scaravilli V, et al. Infections during extracorporeal membrane oxygenation: epidemiology, risk factors, pathogenesis and prevention. Int J Antimicrob Agents. 2017;50:9-16.

25. Venkataraman R, Divatia JV, Ramakrishnan N, et al. Multicenter observational study to evaluate epidemiology and resistance patterns of common intensive care unit-infections. Indian J Crit Care Med. 2018;22:20-6.

26. Alagesan M, Gopalakrishnan R, Panchatcharam SN, Dorairajan S, Mandayam Ananth T, Venkatasubramanian R. A decade of change in susceptibility patterns of Gram- negative blood culture isolates: a single center study. Germs. 2015;5:65-77.

27. Knaus WA, Draper EA, Wagner DP, Zimmerman JE. APACHE II: a severity of disease classification system. Crit Care Med. 1985;13: 818-29.

28. Singer M, Deutschman CS, Seymour CW, et al. The third International Consensus Definitions for Sepsis and Septic Shock (Sepsis-3). JAMA. 2016;315:801-10.

29. Carlet J. Rapid diagnostic methods in the detection of sepsis. Infect Dis Clin N Am. 1999;13:483-94.

30. Kushimoto S, Gando S, Saitoh D, et al. The impact of body temperature abnormalities on the disease severity and outcome in patients with severe sepsis: an analysis from a multicenter, prospective survey of severe sepsis. Crit Care. 2013;17:R271.

31. Hines M, Berkowitz I, Bizzarro M, et al. Infection control and extracorporeal life support. The ELSO Infectious Disease Task Force Recommendations.

32. Steiner CK, Stewart DL, Bond SJ, Hornung CA, McKay VJ. Predictors of acquiring a nosocomial bloodstream infection on extracorporeal membrane oxygenation. J Pediatr Surg. 2001;36:48792.

33. Gendrel D, Bohuon C. Procalcitonin, a marker of bacterial infection. Infection. 1997;25:133-4.

34. Pieri M, Greco T, De Bonis M, et al. Diagnosis of infection in patients undergoing extracorporeal membrane oxygenation: a case-control study. J Thorac Cardiovasc Surg. 2012;143:1411-6.

35. Tanaka D, Pitcher HT, Cavarocchi NC, Hirose H. Can procalcitonin differentiate infection from systemic inflammatory reaction in patients on extracorporeal membrane oxygenation? J Heart Lung Transplant. 2014;33:1186-8.

36. Khan FY. High serum procalcitonin: Interpret with caution. Clin Microbio. 2017;6:e141.

37. Park SY. Procalcitonin to guide antibiotic therapy for critically ill patients in Korea. J Korean Med Sci. 2019;34:e122.

38. Weinstein MP, Reller LB, Murphy JR, Lichtenstein KA. The clinical significance of positive blood cultures: a comprehensive analysis of 500 episodes of bacteremia and fungemia in adults. Rev Infect Dis. 1983;5:35-53.

39. Bansal N, Gopalakrishnan R, Sethuraman N, et al. Experience with $\beta$-D-Glucan Assay in the management of critically ill patients with 
high risk of Invasive Candidiasis: an observational study. Indian J Crit Care Med. 2018;22:364-8.

40. Brown KL, Ridout DA, Shaw M, et al. Healthcare-associated infection in pediatric patients on extracorporeal life support: the role of multidisciplinary surveillance. Pediatr Crit Care Med. 2006;7: $546-50$.

41. de Roux Q, Botterel F, Lepeule R, Taccone FS, Langeron O, Mongardon N. Candida bloodstream infection under veno-arterial ECMO therapy. Crit Care. 2019;23:314.

42. Sherwin J, Heath T, Watt K. Pharmacokinetics and dosing of antiinfective drugs in patients on extracorporeal membrane oxygenation: a review of the current literature. Clin Ther. 2016;38:1976-94.

43. Harthan AA, Buckley KW, Heger ML, Fortuna RS, Mays K. Medication adsorption into contemporary extracorporeal membrane oxygenator circuits. J Pediatr Pharmacol Ther. 2014;4:28895.

44. Shekar K, Roberts JA, Mcdonald CI, et al. Protein-bound drugs are prone to sequestration in the extracorporeal membrane oxygenation circuit: results from an ex vivo study. Crit Care. 2015;19:164.

45. Shekar K, Roberts JA, Mcdonald CI, et al. Sequestration of drugs in the circuit may lead to therapeutic failure during extracorporeal membrane oxygenation. Crit Care. 2012;16:R194.

46. Seghaye MC, Grabitz RG, Duchateau J, et al. Inflammatory reaction and capillary leak syndrome related to cardiopulmonary bypass in neonates undergoing cardiac operations. J Thorac Cardiovasc Surg. 1996;112:687-97.

47. Butler J, Pathi VL, Paton RD, et al. Acute-phase responses to cardiopulmonary bypass in children weighing less than 10 kilograms. Ann Thorac Surg. 1996;62:538-42.

48. Amaker RD, DiPiro JT, Bhatia J. Pharmacokinetics of vancomycin in critically ill infants undergoing extracorporeal membrane oxygenation. Antimicrob Agents Chemother. 1996;40:1139-42.

49. Hoie EB, Swigart SA, Leuschen MP, et al. Vancomycin pharmacokinetics in infants undergoing extracorporeal membrane oxygenation. Clin Pharm. 1990;9:711-5.

50. Boyce JM, Pittet D. Guidelines for hand hygiene in health-care settings: recommendations of the Healthcare Infection Control Practices Advisory Committee and the HICPAC/SHEA/APIC/ IDSA Hand Hygiene Task Force. Infect Control Hosp Epidemiol. 2002;23:S3-S40.

51. Ferrie S, Herkes R, Forrest P. Nutrition support during extracorporeal membrane oxygenation (ECMO) in adults: a retrospective audit of 86 patients. Intensive Care Med. 2013;39:1989-94.

Publisher's note Springer Nature remains neutral with regard to jurisdictional claims in published maps and institutional affiliations. 